\title{
Strongly Convergent Approximations to Fixed Points of Total Asymptotically Nonexpansive Mappings
}

\author{
Yakov Alber \\ Department of Mathematics \\ The Technion-Israel Institute of Technology \\ 32000 Haifa, Israel. Email: alberya@techunix.technion.ac.il \\ Rafa Espínola* and Pepa Lorenzo \\ Departamento de Análisis Matemático \\ Universidad de Sevilla, P.O. Box 1160 \\ 41080-Seville, Spain. Emails: espinola@us.es, ploren@us.es
}

* Corresponding author

\begin{abstract}
In this work we prove a new strong convergence result of the regularized successive approximation method given by

$$
y_{n+1}=q_{n} z_{0}+\left(1-q_{n}\right) T^{n} y_{n}, \quad n=1,2, \ldots
$$

where

$$
\lim _{n \rightarrow \infty} q_{n}=0 \text { and } \sum_{n=1}^{\infty} q_{n}=\infty,
$$

for $T$ a total asymptotically nonexpansive mapping, i.e., $T$ is such that

$$
\left\|T^{n} x-T^{n} y\right\| \leq\|x-y\|+k_{n}^{(1)} \phi(\|x-y\|)+k_{n}^{(2)},
$$

where $k_{n}^{1}$ and $k_{n}^{2}$ are real null convergent sequences and $\phi: \mathbf{R}^{+} \rightarrow \mathbf{R}^{+}$is continuous and such that $\phi(0)=0$ and $\lim _{t \rightarrow \infty} \frac{\phi(t)}{t} \leq C$ for a certain constant $C>0$.

Among other features, our results essentially generalize existing results on strong convergence for $T$ nonexpansive and asymptotically nonexpansive. The convergence and stability analysis is given for both self- and nonself-mappings.
\end{abstract}

RUNNING TITLE: Strong convergence to fixed points

KEY WORDS: asymptotically nonexpansive mappings, best approximation, fixed point, duality map, iteration schemes.

Mathematics Subject Classification 2000: 47A58, 47H10. 


\section{Introduction}

Iterative procedures for nonlinear operators have been largely studied by many authors in the last decades. One of the first results of this nature was obtained by Browder [5] for nonexpansive self-mappings defined on Hilbert spaces. Here Browder studied the iterative method:

$$
x_{\omega}=\omega z_{0}+(1-\omega) T x_{\omega} .
$$

for $\Omega$ a closed and convex subset of $H, z_{0} \in \Omega$ an arbitrary point and $T: \Omega \rightarrow \Omega$ a nonexpansive mapping with nonempty fixed point set $\mathcal{N}(T):=\{x \in \Omega: T x=x\}$.

In [5], Browder proved that $\lim _{\omega \rightarrow 0} x_{\omega}$ exists and is a fixed point of $T$. This result was extended by Reich [17] to the case when $X$ is a uniformly smooth Banach space. Furthermore, he showed that the fixed point set of $T$ is a sunny nonexpansive retract of $\Omega$.

The recursive formula (explicit scheme)

$$
y_{1} \in \Omega, \quad y_{n+1}=q_{n} z_{0}+\left(1-q_{n}\right) T y_{n}, \quad n=1,2, \ldots,
$$

was introduced by Halpern [12] who discussed its convergence in the framework of Hilbert spaces. Later it has been investigated in $[12,18,19,20]$ with different additional properties on the sequence $\left\{q_{n}\right\}$, the operator $T$ and the space $X$.

Browder's and Halpern's iterative procedures have motivated different schemes to find fixed points of asymptotically nonexpansive mappings (see Remark 1.2 for definition). In this way, T.C. Lim and H.K. Xu [15] studied the algorithm for $T$ asymptotically nonexpansive which generates the sequence (implicit scheme)

$$
x_{n}=q_{n} z_{0}+\left(1-q_{n}\right) T^{n} x_{n} .
$$

They showed that the sequence $\left\{x_{n}\right\}$ converges strongly to a fixed point of $T$ in the framework of a uniformly smooth Banach space, under suitable conditions on the coefficients. Very recently, in [6], the strong convergence of the explicit scheme given by

$$
y_{1} \in \Omega \quad y_{n+1}=q_{n} z_{0}+\left(1-q_{n}\right) T^{n} y_{n}, \quad n=1,2, \ldots,
$$

where $z_{0} \in \Omega$, and

$$
\lim _{n \rightarrow \infty} q_{n}=0 \text { and } \sum_{n=1}^{\infty} q_{n}=\infty
$$

has been studied in uniformly smooth spaces. It is worthwhile to point out that the convergence of the implicit scheme given by (1.3) is an important tool in order to prove the strong convergence of explicit schemes as (1.2).

In this paper we will consider the class of the total asymptotically nonexpansive mappings which have been introduced very recently in [2].

Definition 1.1 (cf. [2]) A mapping $T: \Omega \rightarrow \Omega$ is called total asymptotically nonexpansive if there exist nonnegative real sequences $\left\{k_{n}^{(1)}\right\}$ and $\left\{k_{n}^{(2)}\right\}$ with $k_{n}^{(1)}, k_{n}^{(2)} \rightarrow 0$ as $n \rightarrow \infty$, and a continuous function $\phi: R^{+} \rightarrow R^{+}$with $\phi(0)=0$ such that

$$
\left\|T^{n} x-T^{n} y\right\| \leq\|x-y\|+k_{n}^{(1)} \phi(\|x-y\|)+k_{n}^{(2)} .
$$


Remark 1.2 If $\phi(\lambda) \equiv 0$ then (1.6) takes the form

$$
\left\|T^{n} x-T^{n} y\right\| \leq\|x-y\|+k_{n}^{(2)} .
$$

Hence, if $\Omega$ is a bounded set and $T^{N}$ is continuous for some integer $N \geq 1$ the mapping $T$ is of asymptotically nonexpansive type. If $\phi(\lambda)=\lambda$ then we can write

$$
\left\|T^{n} x-T^{n} y\right\| \leq\left(1+k_{n}^{(1)}\right)\|x-y\|+k_{n}^{(2)} .
$$

In addition, if $k_{n}^{(2)}=0$ for all $n \geq 1$ then we obtain the definition of asymptotically nonexpansive mapping:

$$
\left\|T^{n} x-T^{n} y\right\| \leq k_{n}\|x-y\|, \quad k_{n} \rightarrow 1 .
$$

If $k_{n}^{(1)}=0$ and $k_{n}^{(2)}=0$ for all $n \geq 1$ then we obtain the class of nonexpansive mappings:

$$
\|T x-T y\| \leq\|x-y\| .
$$

If $k_{1}^{(2)}=0$ then it follows from (1.6) that $T$ is uniformly continuous, however, it can be uniformly continuous even if $k_{1}^{(2)} \neq 0$.

To construct the strong convergent approximations to solutions of the equation

$$
T x=x
$$

with a total asymptotically nonexpansive mapping $T$, we apply the iterative scheme given by (1.4).

For nonexpansive operators $T$, the algorithm (1.4) is written down in the following form:

$$
y_{1} \in \Omega, \quad y_{n+1}=q_{n} z_{0}+\left(1-q_{n}\right) T y_{n}, \quad n=1,2, \ldots,
$$

where $\lim _{n \rightarrow \infty} q_{n}=0$.

We show next that (1.8) is the regularized successive approximation method for (1.7). As it is known, equation (1.7) is equivalent to

$$
A x=0
$$

with the accretive operator $A=I-T: \Omega \rightarrow \Omega$. That is, in this case

$$
\langle A x-A y, J(x-y)\rangle \geq 0,
$$

where $J$ stands for the normalized duality map. If $x^{*}$ is a solution of (1.7) then $A x^{*}=0$. In the sequel, we assume that the fixed point set $\mathcal{N}(T)$ of $T$ is not empty. We emphasize that the problem (1.7) belongs to the class of ill-posed problems (for more on ill-posted problems see [4]). Strongly convergent approximations to $x^{*}$ can be obtained only by using some regularization procedure.

Let $\omega$ be a parameter such that $0<\omega<1$ and $\omega \rightarrow 1$. Obviously, if $x^{*}$ is a solution of (1.9) then it is solution of the equation

$$
\omega A x=0
$$


for any fixed $\omega>0$. Using the general theory (see for example [4], Section 2.7), construct for (1.11) the operator regularization method with regularization parameter $\alpha=1-\omega \rightarrow 0$, namely,

$$
\omega A x+(1-\omega)\left(x-z_{0}\right)=0,
$$

where $z_{0} \in \Omega$. It is easy to see that (1.12) is equivalent to

$$
x=(1-\omega) z_{0}+\omega T x .
$$

Denote

$$
T_{\omega} x=(1-\omega) z_{0}+\omega T x .
$$

Since $\Omega$ is convex and closed, we have that $T_{\omega}: \Omega \rightarrow \Omega$, and (1.13) can be rewritten as

$$
x=T_{\omega} x .
$$

Consequently, by Banach Contraction Principle, equation (1.12) has a unique solution $x_{\omega}$ and the successive approximation method

$$
x_{1} \in \Omega \quad x_{n+1}=(1-\omega) z_{0}+\omega T x_{n}
$$

converges strongly to $x_{\omega}$. Let $X$ be uniformly smooth and $\omega_{k} \rightarrow 1$ as $k \rightarrow \infty$. Consider now the regularized equation

$$
\omega_{k} A x+\left(1-\omega_{k}\right)\left(x-z_{0}\right)=0
$$

with $k$ fixed and denote by $x_{k}$ its unique solution. Then there exists $\bar{x}^{*} \in \mathcal{N}(T)$ such that $x_{k} \rightarrow \bar{x}^{*}$ as $k \rightarrow \infty$. Moreover (see [4]), $\bar{x}^{*}$ satisfies the inequality

$$
\left\langle\bar{x}^{*}-z_{0}, J\left(\bar{x}^{*}-x^{*}\right)\right\rangle \geq 0 \quad \forall x^{*} \in \mathcal{N}(T) .
$$

It can be shown in the same way that (1.8) with $T^{n}$ in place of $T$ is the regularized successive approximation method for the equation (1.7) with total asymptotically nonexpansive mapping. Indeed, if total asymptotically nonexpansive mappings are considered in place of nonexpansive mappings, then

$$
\left\langle A^{n} x-A^{n} y, J(x-y)\right\rangle \geq-k_{n}^{(1)} \phi(\|x-y\|)\|x-y\|-k_{n}^{(2)}\|x-y\|,
$$

where $A^{n}=I-T^{n}$. It is clear that the analysis of strong convergence is more difficult in this situation, moreover, very little is known about the structure of the solution set. In particular the same holds for asymptotically nonexpansive mappings for which (1.16) is

$$
\left\langle A^{n} x-A^{n} y, J(x-y)\right\rangle \geq-k_{n}^{(1)}\|x-y\|^{2} .
$$

The main result of this paper, Theorem 3.1 in Section 3, states a strong convergence result for the iterative scheme (1.4) in reflexive Banach spaces with a weakly continuous duality map on unbounded domains. Notice that it is an open question wether a reflexive Banach space admitting a weakly sequentially continuous duality mapping is uniformly smooth. An implicit scheme convergence result is also proved. This result is used to guarantee the existence of sunny nonexpansive retractions.

In Section 4 we study the same iterative scheme for total asymptotically nonexpansive nonself-mapping. Finally, in Section 5, our last section we investigate the stability problem for iterative schemes with respect to perturbations of constraint sets for nonexpansive nonself-mappings. 


\section{Preliminaries}

Let $X$ be a real Banach space with norm $\|\cdot\|$, let $X^{*}$ be its dual space with the norm $\|\cdot\|_{*}$ and, as usual, denote the duality pairing of $X$ and $X^{*}$ by $\langle\varphi, x\rangle$, where $x \in X$ and $\varphi \in X^{*}$ (in other words, $\langle\varphi, x\rangle$ is the value of $\varphi$ at $x$ ).

It is said that $X$ is uniformly smooth if for any given $\varepsilon>0$, there exists $\delta>0$ such that for all $x, y \in X$ with $\|x\|=1$ and $\|y\| \leq \delta$, the inequality

$$
2^{-1}(\|x+y\|+\|x-y\|)-1 \leq \varepsilon\|y\|
$$

holds. The function

$$
\rho_{X}(\tau)=\sup \left\{2^{-1}(\|x+y\|+\|x-y\|)-1:\|x\|=1,\|y\|=\tau\right\}
$$

is called the modulus of smoothness of the space $X$.

This function is increasing and approaches to zero as $\tau \rightarrow 0$. Denote $h_{X}(\tau)=\frac{\rho_{X}(\tau)}{\tau}$. Observe that the space $X$ is uniformly smooth if and only if $\lim _{\tau \rightarrow 0} h_{X}(\tau)=0$.

Let $\psi:[0, \infty) \rightarrow[0, \infty)$ be a continuous strictly increasing function such that $\psi(t) \rightarrow \infty$ as $t \rightarrow \infty$ and $\psi(0)=0$. The generalized duality mapping $J_{\psi}: X \rightarrow 2^{X^{*}}$ associated to a gauge function $\psi$ is defined as

$$
J_{\psi}(x)=\left\{x^{*} \in X^{*}:\left\langle x, x^{*}\right\rangle=\psi(\|x\|)\|x\|,\left\|x^{*}\right\|=\psi(\|x\|)\right\}, \quad x \in X .
$$

In the case that $\psi(t)=t$ then $J_{\psi}=J$ which is the normalized duality map.

We say that a Banach space $X$ has a weakly continuous duality map ([5]) if there exists a gauge function $\psi$ for which the generalized duality map $J_{\psi}$ is single-valued and weak-toweak* sequentially continuous.

It is well-known that $J_{\psi}$ is the subdifferential, in the sense of convex analysis, of the convex function

$$
\Phi(t)=\int_{0}^{t} \psi(\tau) d \tau, \text { for } \tau \geq 0
$$

and that $J_{\psi}$ is single-valued if and only if $X$ is smooth. We will need the following subdifferential inequality which is known to hold in smooth spaces:

$$
\Phi(\|x+y\|) \leq \Phi(\|x\|)+\left\langle y, J_{\psi}(x+y)\right\rangle
$$

for any $x, y \in X$.

Next we introduce some definitions and auxiliary results that will be needed in the sequel.

Definition 2.1 Let $X$ be a Banach space and $C$ a nonempty closed convex subset of $X$. An operator $T: C \rightarrow X$ is demiclosed (at $y$ ) if $T(x)=y$ whenever $\left\{x_{n}\right\} \subseteq C$ is a sequence weakly convergent to $x$ and $T\left(x_{n}\right) \rightarrow y$ as $n \rightarrow \infty$. 
Definition 2.2 A Banach space $X$ satisfies the Opial's condition if for each sequence $\left\{x_{n}\right\}$ in $X$, the relation $x_{n} \rightarrow x$ implies that

$$
\limsup _{n \rightarrow \infty}\left\|x_{n}-x\right\|<\limsup _{n \rightarrow \infty}\left\|x_{n}-y\right\|
$$

for all $y \in X$ with $x \neq y$.

Definition 2.3 A Banach space $X$ satisfies the Generalized Gossez-Lami Dozo property (GGLD-property) if

$$
\liminf _{n \rightarrow \infty}\left\|x_{n}\right\|<\limsup _{m \rightarrow \infty} \limsup _{n \rightarrow \infty}\left\|x_{m}-x_{n}\right\|
$$

whenever $\left\{x_{n}\right\}$ is a weak null sequence which is not norm convergent.

The following demiclosedness principle can be found in [11].

Theorem 2.4 Let $X$ be a Banach space with GGLD-property and Opial's condition. Let $C$ be a weakly compact convex subset of $X$ and $T: C \rightarrow C$ a uniformly continuous mapping of asymptotically nonexpansive type. Then $I-T$ is demiclosed at zero.

The following result is well-known (see [13] and [14]).

Proposition 2.5 If in a reflexive Banach space $X$ the duality mapping $J$ is weakly continuous then $X$ satisfies GGLD-property and Opial's condition.

The next corollary follows as a consequence of this proposition and a careful reading of the original proof of Theorem 2.4.

Corollary 2.6 Let $X$ be a reflexive Banach space with a weakly continuous duality mapping $J$. Let $C$ be a closed convex subset of $X$ and $T: C \rightarrow C$ a uniformly continuous mapping and total asymptotically nonexpansive with bounded orbits. Then $I-T$ is demiclosed at zero.

We will also use the concept of a sunny nonexpansive retraction [10] and, in particular, its characterization by means of the duality map in a smooth Banach space.

Definition 2.7 Let $C$ be a non-empty subset of a Banach space $X$ and $D$ a subset of $C$. A mapping $Q: C \rightarrow D$ is said to be

(i) a retraction onto $D$ if $Q^{2}=Q$;

(ii) a nonexpansive retraction if it also satisfies the inequality

$$
\|Q x-Q y\| \leq\|x-y\|, \text { for all } x, y \in C
$$

(iii) a sunny retraction if for all $x \in C$ and for all $0 \leq t<\infty$,

$$
Q(Q x+t(x-Q x))=Q x, \text { whenever } Q x+t(x-Q x) \in C .
$$


Proposition 2.8 Assume that $C$ is a non-empty closed convex subset of a smooth Banach space $X$ and $D$ is a subset of $C$. Then a nonexpansive mapping $Q: C \rightarrow D$ is a sunny retraction if and only if for all $x \in C$ and for all $\xi \in D$,

$$
\langle x-Q x, J(\xi-Q x)\rangle \leq 0 .
$$

In particular, there is at most one sunny nonexpansive retraction on $D$.

Remark 2.9 Proposition 2.5 and Corollary 2.6 remain still valid if the normalized duality mapping $J$ is replaced by the duality mapping $J_{\psi}$ with the gauge function $\psi(t)$. Moreover, we can use $J_{\psi}$ to characterize sunny nonexpansive retractions in a smooth Banach space given by Proposition 2.8.

Let $G_{1}$ and $G_{2}$ be nonempty closed subsets of $X$. The Hausdorff distance between $G_{1}$ and $G_{2}$ is defined by the following formula:

$$
\mathcal{H}\left(G_{1}, G_{2}\right)=\max \left\{\sup _{z_{1} \in G_{1}} \inf _{z_{2} \in G_{2}}\left\|z_{1}-z_{2}\right\|, \sup _{z_{1} \in G_{2}} \inf _{z_{2} \in G_{1}}\left\|z_{1}-z_{2}\right\|\right\}
$$

We need the following lemma [3] in order to prove the main result of Section 5.

Lemma 2.10 If $X$ is a uniformly smooth Banach space, $\Omega_{1}$ and $\Omega_{2}$ are closed convex subsets of $X$ such that the Hausdorff distance $\mathcal{H}\left(\Omega_{1}, \Omega_{2}\right) \leq \sigma$ and $Q_{\Omega_{1}}$ and $Q_{\Omega_{2}}$ are the (unique) sunny nonexpansive retractions onto the subsets $\Omega_{1}$ and $\Omega_{2}$, respectively, then

$$
\left\|Q_{\Omega_{1}} x-Q_{\Omega_{2}} x\right\|^{2} \leq 16 R(2 r+d) h_{X}\left(16 L R^{-1} \sigma\right)
$$

where $r=\|x\|, d=\max \left\{d_{1}, d_{2}\right\}, R=2(2 r+d)+\sigma$ and $1<L<1.7$ is the Figiel constant $[1,2,9]$. Here $d_{i}=\operatorname{dist}\left(\theta, \Omega_{i}\right), i=1,2$, and $\theta$ is the origin of the space $X$.

We will often apply the following lemma on numerical recurrent inequalities.

Lemma 2.11 Let $\left\{\lambda_{n}\right\}$ and $\left\{\gamma_{n}\right\}$ be nonnegative, $\left\{\alpha_{n}\right\}$ be positive real numbers such that

$$
\lambda_{n+1} \leq \lambda_{n}-\alpha_{n} \lambda_{n}+\gamma_{n}, \quad \forall n \geq 1 .
$$

Let for all $n>1$

$$
\frac{\gamma_{n}}{\alpha_{n}} \leq c_{1} \quad \text { and } \quad \alpha_{n} \leq \alpha
$$

Then $\lambda_{n} \leq \max \left\{\lambda_{1}, K_{*}\right\}$, where $K_{*}=(1+\alpha) c_{1}$. In addition, if

$$
\sum_{1}^{\infty} \alpha_{n}=\infty \quad \text { and } \quad \frac{\gamma_{n}}{\alpha_{n}} \rightarrow 0
$$

then $\lambda_{n} \rightarrow 0$ as $n \rightarrow \infty$. 


\section{Convergence Analysis of Successive Approximation Method}

The goal of this section is to prove strong convergence of the regularized successive approximation method (1.4). Let us consider the explicit scheme (1.4) given by

$$
y_{0} \in \Omega, \quad y_{n+1}=q_{n} z_{0}+\left(1-q_{n}\right) T^{n} y_{n}, \quad n=1,2, \ldots,
$$

with

$$
\lim _{n \rightarrow \infty} q_{n}=0 \text { and } \sum_{n=1}^{\infty} q_{n}=\infty
$$

Theorem 3.1 Let $\Omega$ be a nonempty closed and convex subset of a smooth reflexive Banach space $X$ with a weakly sequentially continuous duality map $J_{\psi}, T: \Omega \rightarrow \Omega$ a uniformly continuous mapping which is total asymptotically nonexpansive with nonempty fixed point set $\mathcal{N}(T)$. Let $\mathcal{N}(T)$ be such that there exists a sunny nonexpansive retraction $Q: \Omega \rightarrow \mathcal{N}(T)$.

Let $z_{0} \in \Omega$ and $\left\{q_{n}\right\} \subset(0,1]$ a sequence satisfying (3.1). Let the sequence $\left\{y_{n}\right\}$ be generated by (1.4). Assume that

$$
\lim _{n \rightarrow \infty} \frac{k_{n}^{(1)}+k_{n}^{(2)}}{q_{n}}=0,
$$

and that there exist positive constants $M_{0}$ and $M_{1}$ such that $\phi(\lambda) \leq M_{0} \lambda$ for $\lambda \geq M_{1}$. Suppose that $\lim _{n}\left\|y_{n}-T y_{n}\right\|=0$, then $\left\{y_{n}\right\}$ converges strongly to the fixed point $\bar{x}^{*}=Q z_{0}$ of $T$.

Proof. Firstly we observe that the sequence $\left\{y_{n}\right\} \subset \Omega$ because $\Omega$ is convex. Take $x^{*} \in \mathcal{N}(T)$. It follows from (1.4) that

$$
\begin{gathered}
\left\|y_{n+1}-x^{*}\right\| \leq q_{n}\left\|z_{0}-x^{*}\right\|+\left(1-q_{n}\right)\left\|T^{n} y_{n}-T^{n} x^{*}\right\| \\
\leq q_{n}\left\|z_{0}-x^{*}\right\|+\left(1-q_{n}\right)\left(\left\|y_{n}-x^{*}\right\|+k_{n}^{(1)} \phi\left(\left\|y_{n}-x^{*}\right\|\right)+k_{n}^{(2)}\right) .
\end{gathered}
$$

Denoting $\lambda_{n}=\left\|y_{n}-x^{*}\right\|$ we have

$$
\lambda_{n+1} \leq\left(1-q_{n}\right) \lambda_{n}+q_{n}\left\|z_{0}-x^{*}\right\|+\left(1-q_{n}\right)\left(k_{n}^{(1)} \phi\left(\lambda_{n}\right)+k_{n}^{(2)}\right) .
$$

Since $\phi$ is continuous it attains its maximum $M$ on $\left[0, M_{1}\right]$. Then it is easy to verify that for all $\lambda \in[0, \infty)$

$$
\phi(\lambda) \leq M+M_{0} \lambda
$$

The inequality (3.3) is rewritten as

$$
\lambda_{n+1} \leq \lambda_{n}-\left(q_{n}-\left(1-q_{n}\right) k_{n}^{(1)} M_{0}\right) \lambda_{n}+\gamma_{n},
$$

where

$$
\gamma_{n}=\left(1-q_{n}\right)\left(k_{n}^{(1)} M+k_{n}^{(2)}\right)+q_{n}\left\|z_{0}-x^{*}\right\| .
$$


Without loss of generality, in view of (3.2), we assume that there exist constants $\alpha \in(0,1)$ and $M_{2}>0$ such that for all $n \geq 1$

$$
\frac{k_{n}^{(1)}}{q_{n}} \leq \frac{M_{0}(1-\alpha)}{1-q_{n}},
$$

and

$$
\frac{\gamma_{n}}{q_{n}} \leq \alpha M_{2}
$$

Then

$$
\lambda_{n+1} \leq \lambda_{n}-\alpha q_{n} \lambda_{n}+\gamma_{n}
$$

By Lemma 2.11, we conclude that

$$
\lambda_{n} \leq \max \left\{\lambda_{1},(1+\alpha) M_{2}\right\} .
$$

Thus, the sequence $\left\{y_{n}-x^{*}\right\}$ is bounded, which, clearly, implies that $\left\{y_{n}\right\}$ is a bounded sequence.

Applying the subdifferential inequality to

$$
y_{n+1}-Q z_{0}=\left(1-q_{n}\right)\left(T^{n} y_{n}-Q z_{0}\right)+q_{n}\left(z_{0}-Q z_{0}\right)
$$

we deduce that

$$
\Phi\left(\left\|y_{n+1}-Q z_{0}\right\|\right) \leq \Phi\left(\left(1-q_{n}\right)\left\|T^{n} y_{n}-Q z_{0}\right\|\right)+q_{n}\left\langle z_{0}-Q z_{0}, J_{\psi}\left(y_{n+1}-Q z_{0}\right)\right\rangle .
$$

Since $T$ is total asymptotically nonexpansive and $Q z_{0} \in \mathcal{N}(T)$, we have

$$
\left\|T^{n} y_{n}-Q z_{0}\right\| \leq\left\|y_{n}-Q z_{0}\right\|+\nu_{n}
$$

where $\nu_{n}=M k_{n}^{(1)}+M_{0} k_{n}^{(1)}\left\|y_{n}-Q z_{0}\right\|+k_{n}^{(2)}$ is bounded and vanishes as $n \rightarrow \infty$. Now, since $\Phi$ is a convex and nondecreasing, for $n$ large enough we have

$$
\begin{aligned}
\Phi\left(\left\|T^{n} y_{n}-Q z_{0}\right\|\right) \leq & \left(1-\nu_{n}\right) \Phi\left(\left\|y_{n}-Q z_{0}\right\|\right)+\nu_{n} \Phi\left(\left\|y_{n}-Q z_{0}\right\|+1\right) \\
& \leq \Phi\left(\left\|y_{n}-Q z_{0}\right\|\right)+\nu_{n} M_{3}
\end{aligned}
$$

for $M_{3}$ a suitable constant. Consequently

$$
\begin{gathered}
\Phi\left(\left\|y_{n+1}-Q z_{0}\right\|\right) \leq\left(1-q_{n}\right) \Phi\left(\left\|y_{n}-Q z_{0}\right\|\right)+\left(1-q_{n}\right) \nu_{n} M_{3}+ \\
+q_{n}\left\langle z_{0}-Q z_{0}, J_{\psi}\left(y_{n+1}-Q z_{0}\right)\right\rangle .
\end{gathered}
$$

We claim that

$$
\limsup _{n \rightarrow \infty}\left\langle z_{0}-Q z_{0}, J_{\psi}\left(y_{n}-Q z_{0}\right)\right\rangle \leq 0 .
$$

Indeed, since the sequence $\left\{y_{n}\right\}$ is bounded and the space $X$ reflexive there exists a subsequence $\left\{y_{n_{k}}\right\}$ which is weakly convergent in $\Omega$. Let $\bar{y}$ be its weak limit. We can fix this subsequence so that

$$
\limsup _{n \rightarrow \infty}\left\langle z_{0}-Q z_{0}, J_{\psi}\left(y_{n}-Q z_{0}\right)\right\rangle=\lim _{k \rightarrow \infty}\left\langle z_{0}-Q z_{0}, J_{\psi}\left(y_{n_{k}}-Q z_{0}\right)\right\rangle
$$


But we know that $y_{n_{k}}-T y_{n_{k}} \rightarrow 0$ as $k \rightarrow \infty$, so, from the demiclosedness principle, we have that $\bar{y}$ is a fixed point of $T$. From the weak continuity of $J_{\psi}$ and the characterization of sunny nonexpansive retraction (Proposition 2.8), our claim follows in the following way

$$
\limsup _{n \rightarrow \infty}\left\langle z_{0}-Q z_{0}, J_{\psi}\left(y_{n}-Q z_{0}\right)\right\rangle=\left\langle z_{0}-Q z_{0}, J_{\psi}\left(\bar{y}-Q z_{0}\right)\right\rangle \leq 0 .
$$

Let us consider now (3.5), which we rewrite as follows

$$
\lambda_{n+1} \leq \lambda_{n}-q_{n} \lambda_{n}+\gamma_{n}^{\prime}
$$

where $\lambda_{n}=\Phi\left(\left\|y_{n}-Q z_{0}\right\|\right)$ and

$$
\gamma_{n}^{\prime}=q_{n}\left\langle z_{0}-Q z_{0}, J_{\psi}\left(y_{n+1}-Q z_{0}\right)\right\rangle+\left(1-q_{n}\right) \nu_{n} M_{3} .
$$

If we make $\alpha_{n}=q_{n}$ and $\gamma_{n}=\max \left\{0, \gamma_{n}^{\prime}\right\}$, we can apply Lemma 2.11 to deduce that $\lambda_{n} \rightarrow 0$ as $n \rightarrow \infty$. Therefore $\left\{y_{n}\right\}$ converges strongly to $Q z_{0}$ and the proof is complete.

Next we study different situations that guarantee the fulfillment of some of the conditions imposed in Theorem 3.1. Observe first that if $\left\{y_{n}\right\}$ has a limit then

$$
\lim _{n \rightarrow \infty}\left\|y_{n+1}-y_{n}\right\|=0
$$

Lemma 3.2 Condition (3.6) is sufficient to guarantee $\lim _{n}\left\|y_{n}-T y_{n}\right\|=0$ in the previous theorem.

Proof. Following the proof of Theorem 3.1, the sequence $\left\{y_{n}-x^{*}\right\}$ is bounded, say $\left\|y_{n}-x^{*}\right\| \leq C_{1}$. It is clear that

$$
\left\|y_{n}\right\| \leq\left\|y_{n}-x^{*}\right\|+\left\|x^{*}\right\| \leq C_{1}+\left\|x^{*}\right\|=C .
$$

Observe that if $C_{1} \leq M_{1}$ then $\phi\left(\left\|y_{n}-x^{*}\right\|\right) \leq M$. At the same time, if $C_{1} \geq M_{1}$ then $\phi\left(\left\|y_{n}-x^{*}\right\|\right) \leq M_{0}\left\|y_{n}-x^{*}\right\| \leq M_{0} C_{1}$. Therefore,

$$
\phi\left(\left\|y_{n}-x^{*}\right\|\right) \leq \max \left\{M, M_{0} C_{1}\right\}=\bar{M} .
$$

In addition,

$$
\begin{gathered}
\left\|T^{n} y_{n}\right\| \leq\left\|T^{n} y_{n}-T^{n} x^{*}\right\|+\left\|x^{*}\right\| \\
\leq\left\|y_{n}-x^{*}\right\|+k_{n}^{(1)} \phi\left(\left\|y_{n}-x^{*}\right\|\right)+k_{n}^{(2)}+\left\|x^{*}\right\| .
\end{gathered}
$$

This means that the sequence $\left\{T^{n} y_{n}\right\}$ is bounded too. Then algorithm (1.4) yields the following limit equality:

$$
\lim _{n \rightarrow \infty}\left(y_{n+1}-T^{n} y_{n}\right)=\lim _{n \rightarrow \infty}\left(q_{n}\left(z_{0}-T^{n} y_{n}\right)\right)=0 .
$$

Since

$$
\left\|y_{n+1}-T^{n} y_{n+1}\right\| \leq\left\|y_{n+1}-T^{n} y_{n}\right\|+\left\|T^{n} y_{n}-T^{n} y_{n+1}\right\|
$$


we deduce that

$$
\left\|y_{n+1}-T^{n} y_{n+1}\right\| \leq\left\|y_{n+1}-T^{n} y_{n}\right\|+\left\|y_{n}-y_{n+1}\right\|+k_{n}^{(1)} \phi\left(\left\|y_{n}-y_{n+1}\right\|\right)+k_{n}^{(2)} .
$$

In addition, from (3.8) and the fact that, by hypothesis, $\left\|y_{n+1}-y_{n}\right\| \rightarrow 0$ as $n \rightarrow \infty$, we obtain that

$$
\lim _{n \rightarrow \infty}\left(y_{n}-T^{n-1} y_{n}\right)=0 .
$$

Now,

$$
\left\|y_{n}-T y_{n}\right\| \leq\left\|y_{n}-T^{n} y_{n}\right\|+\left\|T^{n} y_{n}-T y_{n}\right\|
$$

In view of (3.8),

$$
\left\|T^{n} y_{n}-y_{n}\right\| \leq\left\|T^{n} y_{n}-y_{n+1}\right\|+\left\|y_{n+1}-y_{n}\right\| \rightarrow 0 \quad \text { as } n \rightarrow \infty .
$$

Since $T$ is uniformly continuous, there exists a continuous increasing function $\omega: \mathbf{R} \rightarrow \mathbf{R}$ with $\omega(0)=0$ satisfying the relations

$$
\left\|T^{n} y_{n}-T y_{n}\right\|=\left\|T\left(T^{n-1} y_{n}\right)-T y_{n}\right\| \leq \omega\left(\left\|T^{n-1} y_{n}-y_{n}\right\|\right) .
$$

By (3.9), it is easy to see that

$$
\left\|T^{n} y_{n}-T y_{n}\right\| \rightarrow 0 \quad \text { as } n \rightarrow \infty .
$$

Now (3.10) and (3.11) complete the proof.

The next two propositions give conditions for the fulfillment of condition (3.6).

Proposition 3.3 Let $\Omega$ be a closed convex subset of a Banach space $X, T: \Omega \rightarrow \Omega$ a total asymptotically nonexpansive mapping with nonempty fixed point set $\mathcal{N}(T)$. Take $z_{0}$ in $\Omega$, $x^{*} \in \mathcal{N}(T)$ and $\left\{q_{n}\right\}$ a decreasing sequence in $(0,1)$ satisfying (3.1). Let the sequence $\left\{y_{n}\right\}$ be generated by (1.4). If the sequence $\left\{T^{n} y_{n}\right\}$ is bounded and

$$
\lim _{n \rightarrow \infty}\left\|T^{n} y_{n}-T^{n-1} y_{n-1}\right\|=0
$$

then (3.6) holds.

Proof. We have from (1.4)

$$
y_{n+1}-y_{n}=\left(q_{n}-q_{n-1}\right) z_{0}+\left(1-q_{n}\right) T^{n} y_{n}-\left(1-q_{n-1}\right) T^{n-1} y_{n-1} .
$$

Then

$$
\left\|y_{n+1}-y_{n}\right\| \leq\left|q_{n}-q_{n-1}\right|\left(\left\|z_{0}\right\|+\left\|T^{n} y_{n}\right\|\right)+\left(1-q_{n}\right)\left\|T^{n} y_{n}-T^{n-1} y_{n-1}\right\| .
$$

The assumptions of the Proposition imply the claim. 
Proposition 3.4 Let $\Omega$ be a closed convex subset of a Banach space $X, T: \Omega \rightarrow \Omega$ a total asymptotically nonexpansive mapping with a nonempty fixed point set $\mathcal{N}(T)$. Take $z_{0}$ some point in $\Omega, x^{*} \in \mathcal{N}(T)$ and $\left\{q_{n}\right\}$ a decreasing sequence in $(0,1)$ satisfying (3.1). Let the sequence $\left\{y_{n}\right\}$ be generated by (1.4). If (3.2) holds, there exist positive constants $M_{0}$ and $M_{1}$ such that $\phi(\lambda) \leq M_{0} \lambda$ for $\lambda \geq M_{1}$, and, if additionally, we assume that $\lim \frac{q_{n-1}}{q_{n}}$ exists and

$$
\lim _{n \rightarrow \infty} \frac{\left\|T^{n} y_{n-1}-T^{n-1} y_{n-1}\right\|}{q_{n}}=0
$$

then (3.6) holds.

Proof. It is not difficult to state the following difference:

$$
\begin{gathered}
y_{n+1}-y_{n}=\left(1-q_{n}\right)\left(T^{n} y_{n}-T^{n} y_{n-1}\right)+\left(q_{n}-q_{n-1}\right)\left(z_{0}-x^{*}\right) \\
+\left(q_{n-1}-q_{n}\right)\left(T^{n-1} y_{n-1}-T^{n} x^{*}\right)+\left(1-q_{n}\right)\left(T^{n} y_{n-1}-T^{n-1} y_{n-1}\right) .
\end{gathered}
$$

We have

$$
\left\|T^{n} y_{n}-T^{n} y_{n-1}\right\| \leq\left\|y_{n}-y_{n-1}\right\|+k_{n}^{(1)} M+k_{n}^{(1)} M_{0}\left\|y_{n}-y_{n-1}\right\|+k_{n}^{(2)} .
$$

Further,

$$
\begin{gathered}
\left\|\left(q_{n}-q_{n-1}\right)\left(z_{0}-x^{*}\right)+\left(q_{n-1}-q_{n}\right)\left(T^{n-1} y_{n-1}-T^{n-1} x^{*}\right)\right\| \\
\leq\left|q_{n}-q_{n-1}\right|\left(\left\|z_{0}-x^{*}\right\|+\left\|y_{n-1}-x^{*}\right\|+k_{n-1}^{(1)} M+k_{n-1}^{(1)} M_{0}\left\|y_{n-1}-x^{*}\right\|+k_{n-1}^{(2)}\right) .
\end{gathered}
$$

Let

$$
\mu_{n}=\left\|z_{0}-x^{*}\right\|+\left\|y_{n}-x^{*}\right\|+k_{n}^{(1)} M+k_{n}^{(1)} M_{0}\left\|y_{n}-x^{*}\right\|+k_{n}^{(2)} .
$$

Since, by Theorem 3.1, $\left\{y_{n}\right\}$ is bounded, there exists a constant $M_{4}>0$ such that $\mu_{n} \leq M_{4}$ for all $n \geq 1$. Then, since in (3.4) we can chose $\alpha$ so close to 1 as needed,

$$
\begin{aligned}
\left\|y_{n+1}-y_{n}\right\| \leq & \left\|y_{n}-y_{n-1}\right\|-k q_{n}\left\|y_{n}-y_{n-1}\right\|+\left(1-q_{n}\right)\left(k_{n}^{(1)} M+k_{n}^{(2)}\right) \\
& +\left|q_{n}-q_{n-1}\right| M_{4}+\left\|T^{n} y_{n-1}-T^{n-1} y_{n-1}\right\|
\end{aligned}
$$

for a certain positive constant $k$. Denoting $\lambda_{n}=\left\|y_{n}-y_{n-1}\right\|$ one gets

$$
\lambda_{n+1} \leq \lambda_{n}-k q_{n} \lambda_{n}+\left(1-q_{n}\right)\left(k_{n}^{(1)} M+k_{n}^{(2)}\right)+\left|q_{n}-q_{n-1}\right| M_{4}+\left\|T^{n} y_{n-1}-T^{n-1} y_{n-1}\right\| .
$$

Since $\lim \frac{q_{n-1}}{q_{n}}$ exists and $\sum_{1}^{\infty} q_{n}=\infty$ we conclude that $\lim \frac{q_{n-1}}{q_{n}}=1$ and so

$$
\lim \frac{\left|q_{n}-q_{n-1}\right|}{q_{n}}=0
$$

Now (3.2), (3.12), (3.13) and Lemma 2.11 complete the proof.

The second part of this section is devoted to the study of the convergence of the implicit scheme (1.3) for asymptotically nonexpansive mappings and the existence of sunny nonexpansive retractions as those in Theorem 3.1. 
Theorem 3.5 Let $X$ be a smooth reflexive Banach space which has a weakly sequentially continuous duality map $J_{\psi}$ associated to a gauge function $\psi$, let $\Omega$ be a nonempty closed and convex subset of $X$, and $T: \Omega \rightarrow \Omega$ an asymptotically nonexpansive mapping with nonempty fixed point set $\mathcal{N}(T)$. Let $z_{0} \in \Omega$ and $\left\{q_{n}\right\} \subseteq(0,1]$ be a sequence such that $\lim _{n} q_{n}=0$ and $\lim _{n} \frac{k_{n}^{(1)}}{q_{n}}=0$. Then,

(i) for $n \in \mathbf{N}$ large enough, there is a unique $x_{n} \in \Omega$ such that

$$
x_{n}=q_{n} z_{0}+\left(1-q_{n}\right) T^{n} x_{n} .
$$

If additionally we suppose that $\lim _{n \rightarrow \infty}\left\|x_{n}-T x_{n}\right\|=0$, then

(ii) the sequence $\left\{x_{n}\right\}$ strongly converges to a fixed point of $T$ and $\mathcal{N}(T)$ is a sunny nonexpansive retract of $\Omega$.

Proof. It is not hard to check that for $n$ large enough the mapping $T_{n} x=q_{n} z_{0}+$ $\left(1-q_{n}\right) T^{n} x$ is a contraction. Therefore the Banach Contraction Principle implies that the sequence $\left\{x_{n}\right\}$ is well-defined. Next we show that this sequence is bounded. Let $x^{*} \in \mathcal{N}(T)$, then

$$
\begin{gathered}
\left\|x_{n}-x^{*}\right\|=\left\|q_{n}\left(z_{0}-x^{*}\right)+\left(1-q_{n}\right)\left(T^{n} x_{n}-x^{*}\right)\right\| \leq q_{n}\left\|z_{0}-x^{*}\right\|+\left(1-q_{n}\right)\left\|T^{n} x_{n}-x^{*}\right\| \\
\leq q_{n}\left\|z_{0}-x^{*}\right\|+\left(1-q_{n}\right)\left(1+k_{n}^{(1)}\right)\left\|x_{n}-x^{*}\right\| .
\end{gathered}
$$

Henceforth

$$
\left\|x_{n}-x^{*}\right\| \leq \frac{q_{n}}{q_{n}-\left(1-q_{n}\right) k_{n}^{(1)}}\left\|z_{0}-x^{*}\right\| .
$$

The boundedness of $\left\{x_{n}\right\}$ follows from the condition $\lim _{n \rightarrow \infty} \frac{k_{n}^{(1)}}{q_{n}}=0$. Since $X$ is reflexive, there exists a weakly convergent subsequence $\left\{x_{n_{k}}\right\}$ of the sequence $\left\{x_{n}\right\}$. Let $\bar{y} \in \Omega$ its weak limit, then the demiclosedness principle implies that $\bar{y}=T \bar{y}$. Further we will see that $\left\{x_{n_{k}}\right\}$ strongly converges to $\bar{y}$. Indeed,

$$
x_{n_{k}}-\bar{y}=q_{n_{k}}\left(z_{0}-\bar{y}\right)+\left(1-q_{n_{k}}\right)\left(T^{n_{k}} x_{n_{k}}-\bar{y}\right),
$$

from the subdifferential inequality, we obtain

$$
\Phi\left(\left\|x_{n_{k}}-\bar{y}\right\|\right) \leq \Phi\left(\left(1-q_{n_{k}}\right)\left\|T^{n_{k}} x_{n_{k}}-\bar{y}\right\|\right)+q_{n_{k}}\left\langle z_{0}-\bar{y}, J_{\psi}\left(x_{n_{k}}-\bar{y}\right)\right\rangle .
$$

On the other hand,

$$
\Phi\left(\left(1-q_{n_{k}}\right)\left\|T^{n_{k}} x_{n_{k}}-\bar{y}\right\|\right) \leq \Phi\left(\left(1-q_{n_{k}}\right)\left(1+k_{n_{k}}^{(1)}\right)\left\|x_{n_{k}}-\bar{y}\right\|\right) .
$$

Since $\left(1-q_{n_{k}}\right)\left(1+k_{n_{k}}^{(1)}\right)<1$ for $n_{k}$ large enough, we also have from the convexity of the function $\Phi$,

$$
\Phi\left(\left(1-q_{n_{k}}\right)\left\|T^{n_{k}} x_{n_{k}}-\bar{y}\right\|\right) \leq\left(1-q_{n_{k}}\right)\left(1+k_{n_{k}}^{(1)}\right) \Phi\left(\left\|x_{n_{k}}-\bar{y}\right\|\right) .
$$


Hence

$$
\left(q_{n_{k}}+q_{n_{k}} k_{n_{k}}^{(1)}-k_{n_{k}}^{(1)}\right) \Phi\left(\left(\left\|x_{n_{k}}-\bar{y}\right\|\right) \leq q_{n_{k}}\left\langle z_{0}-\bar{y}, J_{\psi}\left(x_{n_{k}}-\bar{y}\right)\right\rangle .\right.
$$

Now, since $x_{n_{k}} \rightarrow \bar{y}$ and $J_{\psi}$ is $\mathrm{w}-\mathrm{w}^{*}$ continuous,

$$
\left\langle z_{0}-\bar{y}, J_{\psi}\left(x_{n_{k}}-\bar{y}\right)\right\rangle \rightarrow 0 \quad \text { as } \quad k \rightarrow \infty .
$$

It is easy to check that $\frac{q_{n_{k}}}{q_{n_{k}}+q_{n_{k}} k_{n_{k}}^{(1)}-k_{n_{k}}^{(1)}} \rightarrow 1$ as $k \rightarrow \infty$, so taking limit in (3.15),

$$
\lim _{k \rightarrow \infty} \Phi\left(\left\|x_{n_{k}}-\bar{y}\right\|\right) \leq 0,
$$

the continuity of $\Phi$ finally implies that $x_{n_{k}} \rightarrow \bar{y}$.

Next we show that $\left\{x_{n}\right\}$ is convergent. Let $x_{n_{k}} \rightarrow z$ and $x_{n_{p}} \rightarrow z^{\prime}$, we will see that $z=z^{\prime}$. Let $x^{*} \in \mathcal{N}(T)$, then

$$
\begin{gathered}
\left\langle x_{n}-T^{n} x_{n}, J_{\psi}\left(x_{n}-x^{*}\right)\right\rangle=\left\langle x_{n}-x^{*}, J_{\psi}\left(x_{n}-x^{*}\right)\right\rangle+\left\langle x^{*}-T^{n} x_{n}, J_{\psi}\left(x_{n}-x^{*}\right)\right\rangle \geq \\
\geq\left\|x_{n}-x^{*}\right\| \psi\left(\left\|x_{n}-x^{*}\right\|\right)-\left\|x^{*}-T^{n} x_{n}\right\|\left\|J_{\psi}\left(x_{n}-x^{*}\right)\right\|_{*} \\
\geq\left\|x_{n}-x^{*}\right\| \psi\left(\left\|x_{n}-x^{*}\right\|\right)-\left(1+k_{n}^{(1)}\right)\left\|x_{n}-x^{*}\right\| \psi\left(\left\|x_{n}-x^{*}\right\|\right) \\
=-k_{n}^{(1)}\left\|x_{n}-x^{*}\right\| \psi\left(\left\|x_{n}-x^{*}\right\|\right) .
\end{gathered}
$$

Since $\left\|x_{n}-x^{*}\right\|$ is bounded and

$$
x_{n}-T^{n} x_{n}=\frac{q_{n}}{1-q_{n}}\left(z_{0}-x_{n}\right)
$$

we have that

$$
\left\langle z_{0}-x_{n}, J_{\psi}\left(x_{n}-x^{*}\right)\right\rangle \geq 0,
$$

where $K$ is a positive number. Then, since $z$ and $z^{\prime}$ are both fixed points of $T$, one gets

$$
\limsup _{p \rightarrow \infty}\left\langle x_{n_{p}}-z_{0}, J_{\psi}\left(x_{n_{p}}-z\right)\right\rangle \leq 0 \text { and } \limsup _{k \rightarrow \infty}\left\langle x_{n_{k}}-z_{0}, J_{\psi}\left(x_{n_{k}}-z^{\prime}\right)\right\rangle \leq 0 .
$$

Now, since $J_{\psi}$ is w-w* continuous, we may conclude that

$$
\left\langle z-z^{\prime}, J_{\psi}\left(z-z^{\prime}\right)\right\rangle \leq 0
$$

and so $z=z^{\prime}$.

We will finish the proof if we show that the mapping $Q$, defined as $Q z_{0}:=\lim _{n} x_{n}$, is a sunny nonexpansive retraction from $\Omega$ onto $\mathcal{N}(T)$. Indeed, we know that if $x^{*} \in \mathcal{N}(T)$ then

$$
\left\langle x_{n}-z_{0}, J_{\psi}\left(x_{n}-x^{*}\right)\right\rangle \leq \frac{\left(1-q_{n}\right) k_{n}^{(1)}}{q_{n}}\left\|x_{n}-x^{*}\right\| \phi\left(\left\|x_{n}-x^{*}\right\|\right) .
$$

Taking limit it follows that

$$
\left\langle Q z_{0}-z_{0}, J_{\psi}\left(Q z_{0}-x^{*}\right)\right\rangle \leq 0,
$$

which states, by Proposition 2.8, that $Q$ is as required. 
Remark 3.6 If $\Omega$ is bounded in the previous theorem then $\mathcal{N}(T) \neq \emptyset[15]$.

Remark 3.7 In [6] similar results to Theorems 3.1 and 3.5 are stated, however techniques are different from those used in this section. Moreover we do not require the domain $\Omega$ to be bounded.

Remark 3.8 The existence of the sunny nonexpansive retraction in Theorem 3.1 is not guaranteed in general. Theorem 3.5 implies however that such a retraction exists if the mapping is asymptotically nonexpansive. Moreover if $\Omega$ is supposed to be bounded and $\mathcal{N}(T) \neq \emptyset$, then there exists a nonexpansive retraction from $\Omega$ onto $\mathcal{N}(T)$ (see [8]).

Remark 3.9 It is worthwhile to note that when $T$ is a nonexpansive mapping and $T^{n}$ is replaced by T, Theorem 3.5 coincides with a result in a recent paper by H.K. Xu in [21]. The convergence of the explicit scheme in Theorem 3.1 seems to be new even for the case of nonexpansive mappings.

\section{Successive Approximation Method for Nonself-mappings}

We consider now the case of total asymptotically nonexpansive nonself-mappings $T: \Omega \rightarrow$ $X$. In place of (1.4), we investigate the iterative process in the form

$$
y_{1} \in \Omega \quad y_{n+1}=q_{n} z_{0}+\left(1-q_{n}\right)\left(Q_{\Omega} T\right)^{n} y_{n}, \quad n=1,2, \ldots
$$

where $Q_{\Omega}$ is a nonexpansive retraction of $X$ onto $\Omega$. A modification on the definition of total asymptotically nonexpansive mapping is, however, needed.

Definition 4.1 (cf. $[2,7]$ ) An operator $T: \Omega \rightarrow X$ is said to be total asymptotically nonexpansive if there exist a nonexpansive retraction $Q_{\Omega}: X \rightarrow \Omega$, nonnegative real sequences $\left\{k_{n}^{(1)}\right\}$ and $\left\{k_{n}^{(2)}\right\}$ with $k_{n}^{(1)}, k_{n}^{(2)} \rightarrow 0$ as $n \rightarrow \infty$, and a continuous functions $\phi: R^{+} \rightarrow R^{+}$ with $\phi(0)=0$ such that

$$
\left\|T\left(Q_{\Omega} T\right)^{n-1} Q_{\Omega} x-T\left(Q_{\Omega} T\right)^{n-1} Q_{\Omega} y\right\| \leq\|x-y\|+k_{n}^{(1)} \phi(\|x-y\|)+k_{n}^{(2)} .
$$

Theorem 4.2 Let $\Omega$ be a closed convex subset of a smooth reflexive Banach space $X$ with a weakly sequentially continuous duality map $J_{\psi}, T: \Omega \rightarrow X$ a total asymptotically nonexpansive mapping with nonempty fixed point set $\mathcal{N}(T)$. Under the conditions of Theorem 3.1 replacing $\lim _{n \rightarrow \infty}\left\|y_{n}-T y_{n}\right\|=0$ by $\lim _{n \rightarrow \infty}\left\|y_{n}-y_{n+1}\right\|=0$, the sequence $\left\{y_{n}\right\}$ generated by (4.1) strongly converges to the fixed point $\bar{x}^{*}=Q z_{0}$ given that there exists a sunny nonexpansive retraction $Q: \Omega \rightarrow \mathcal{N}\left(Q_{\Omega} T\right)$. 
Proof. First of all, we note that $\mathcal{N}\left(Q_{\Omega} T\right)$ is non-empty because $\mathcal{N}(T) \subseteq \mathcal{N}\left(Q_{\Omega} T\right)$. We show next that $\left\{y_{n}\right\} \subseteq \Omega$ is bounded. Take $x^{*} \in \mathcal{N}(T)$. In view of the inequalities

$$
\begin{gathered}
\left\|y_{n+1}-x^{*}\right\| \leq q_{n}\left\|z_{0}-x^{*}\right\|+\left(1-q_{n}\right)\left\|\left(Q_{\Omega} T\right)^{n} y_{n}-\left(Q_{\Omega} T\right)^{n} x^{*}\right\| \\
\leq q_{n}\left\|z_{0}-x^{*}\right\|+\left(1-q_{n}\right)\left\|Q_{\Omega} T\left(Q_{\Omega} T\right)^{n-1} y_{n}-Q_{\Omega} T\left(Q_{\Omega} T\right)^{n-1} x^{*}\right\| \\
\quad \leq q_{n}\left\|z_{0}-x^{*}\right\|+\left(1-q_{n}\right)\left\|T\left(Q_{\Omega} T\right)^{n-1} y_{n}-T\left(Q_{\Omega} T\right)^{n-1} x^{*}\right\| \\
\leq q_{n}\left\|z_{0}-x^{*}\right\|+\left(1-q_{n}\right)\left(\left\|y_{n}-x^{*}\right\|+k_{n}^{(1)} \phi\left(\left\|y_{n}-x^{*}\right\|\right)+k_{n}^{(2)}\right),
\end{gathered}
$$

we conclude, by analogy with Theorem 3.1, that there exist positive constants $C, C_{1}$ and $\bar{M}$ such that $\left\|y_{n}\right\| \leq C,\left\|y_{n}-x^{*}\right\| \leq C_{1}$ and $\phi\left(\left\|y_{n}-x^{*}\right\|\right) \leq \bar{M}$. Since $Q_{\Omega}$ is a nonexpansive mapping, it is not difficult to verify that there exists a constant $\bar{C}>0$ such that $\left\|\left(Q_{\Omega} T\right)^{n} y_{n}\right\| \leq \bar{C}$.

It follows from (4.1) that

$$
\lim _{n \rightarrow \infty}\left(y_{n+1}-\left(Q_{\Omega} T\right)^{n} y_{n}\right)=0 .
$$

On the other hand, we can show that

$$
\lim _{n \rightarrow \infty}\left(y_{n+1}-\left(Q_{\Omega} T\right)^{n} y_{n+1}\right)=0 .
$$

Indeed,

$$
\left\|y_{n+1}-\left(Q_{\Omega} T\right)^{n} y_{n+1}\right\| \leq\left\|y_{n+1}-\left(Q_{\Omega} T\right)^{n} y_{n}\right\|+\left\|\left(Q_{\Omega} T\right)^{n} y_{n}-\left(Q_{\Omega} T\right)^{n} y_{n+1}\right\| .
$$

Due to the total asymptotical nonexpansiveness of $T$, we obtain

$$
\begin{gathered}
\left\|\left(Q_{\Omega} T\right)^{n} y_{n}-\left(Q_{\Omega} T\right)^{n} y_{n+1}\right\| \leq\left\|T\left(Q_{\Omega} T\right)^{n-1} y_{n}-T\left(Q_{\Omega} T\right)^{n-1} y_{n+1}\right\| \\
\leq\left\|y_{n}-y_{n+1}\right\|+k_{n}^{(1)} \phi\left(\left\|y_{n}-y_{n+1}\right\|\right)+k_{n}^{(2)} .
\end{gathered}
$$
(4.4).

Now the boundedness of $\left\{\left(Q_{\Omega} T\right)^{n} y_{n}\right\}$, the fact that $\left\|y_{n}-y_{n+1}\right\| \rightarrow 0$ and (4.3) prove

Further, we have

$$
\left\|y_{n}-Q_{\Omega} T y_{n}\right\| \leq\left\|y_{n}-y_{n+1}\right\|+\left\|y_{n+1}-\left(Q_{\Omega} T\right)^{n} y_{n}\right\|+\left\|\left(Q_{\Omega} T\right)^{n} y_{n}-Q_{\Omega} T y_{n}\right\| .
$$

From the uniform continuity of $T$, we estimate the last term in the form:

$$
\left\|Q_{\Omega} T\left(Q_{\Omega} T\right)^{n-1} y_{n}-Q_{\Omega} T y_{n}\right\| \leq\left\|T\left(Q_{\Omega} T\right)^{n-1} y_{n}-T y_{n}\right\| \leq \omega\left(\left\|\left(Q_{\Omega} T\right)^{n-1} y_{n}-y_{n}\right\|\right) .
$$

By (4.3) and (4.4), one gets

$$
\lim \left(y_{n}-Q_{\Omega} T y_{n}\right)=0
$$


Notice that $Q_{\Omega} T$ is a mapping as required in Theorem 3.1 and then we can apply Theorem 3.1 to the sequence $\left\{y_{n}\right\}$. Indeed, we write

$$
y_{n+1}-Q z_{0}=\left(1-q_{n}\right)\left(\left(Q_{\Omega} T\right)^{n} y_{n}-Q z_{0}\right)+q_{n}\left(z_{0}-Q z_{0}\right) .
$$

we use the subdifferential inequality as in Theorem 3.1, and the rest of the proof follows the pattern with the only difference that we obtain that $\left\{y_{n}\right\}$ strongly converges to $Q z_{0}=\bar{x}^{*}$ which is a fixed point of $Q_{\Omega} T$.

\section{$5 \quad$ Stability Analysis for Nonexpansive Nonself-mappings}

Next we study the stability problem for iterative processes with respect to perturbations of constraint sets. Specifically, we consider a process which involves nonexpansive nonselfmappings in the following form:

$$
y_{1} \in \Omega_{1}, \quad y_{n+1}=q_{n} z_{0}+\left(1-q_{n}\right) Q_{\Omega_{n+1}} T y_{n}, \quad n=1,2, \ldots,
$$

where $Q_{\Omega_{n}}: X \rightarrow \Omega_{n}$ is a sunny nonexpansive retraction, and the proximity between the original set $\Omega$ and $\Omega_{n}$ with $n=1,2, \ldots$ is given by the Hausdorff distance:

$$
\mathcal{H}\left(\Omega_{n}, \Omega\right) \leq \sigma_{n}
$$

Let $G=\cap_{n} \Omega_{n}$ and $\bar{G}=\Omega \cap G \neq \emptyset$.

Theorem 5.1 Let $X$ be a uniformly smooth Banach space which has a weakly sequentially continuous duality map $J_{\psi}$. Assume that $D \subset X$ is a closed convex set, $\Omega \subset D$ and $\Omega_{n} \subset D, n=1,2, \ldots$ are closed convex subsets of $X$ with property (5.2). Let $T: D \rightarrow X$ be a nonexpansive mapping with fixed point set $\mathcal{N}(T)$ such that $\mathcal{N}(T) \cap \Omega \neq \emptyset$. Take $z_{0}$ some point in $\bar{G}, x^{*} \in \mathcal{N}(T) \cap \Omega,\left\{q_{n}\right\}$ a sequence in $(0,1)$ satisfying (3.1) and a non-increasing sequence $\sigma_{n} \leq \sigma$, such that $\sigma_{n} \rightarrow 0$ as $n \rightarrow \infty$. Let the sequence $\left\{y_{n}\right\}$ be generated by (5.1). We suppose that the sequence $\left\{T y_{n}\right\}$ is bounded,

$$
\lim _{n \rightarrow \infty} \frac{\left|q_{n}-q_{n-1}\right|}{q_{n}}=0
$$

and

$$
\lim _{n \rightarrow \infty} \frac{\sqrt{h_{X}\left(\sigma_{n}\right)}}{q_{n}}=0 .
$$

Then $\left\{y_{n}\right\}$ converges strongly to the fixed point $\bar{x}^{*}=Q z_{0}$ of $Q_{\Omega} T$, where $Q: \Omega \rightarrow \mathcal{N}\left(Q_{\Omega} T\right)$ is the unique sunny nonexpansive retraction onto $\mathcal{N}\left(Q_{\Omega} T\right)$.

Proof. We show first that $\left\{y_{n}\right\}$ is bounded. It is not difficult to see that

$$
\begin{gathered}
\left\|y_{n+1}-x^{*}\right\|=\left\|q_{n} z_{0}+\left(1-q_{n}\right) Q_{\Omega} T y_{n}-Q_{\Omega} T x^{*}+\left(1-q_{n}\right)\left(Q_{\Omega_{n+1}} T y_{n}-Q_{\Omega} T y_{n}\right)\right\| \\
\leq q_{n}\left\|z_{0}-x^{*}\right\|+\left(1-q_{n}\right)\left\|Q_{\Omega} T y_{n}-Q_{\Omega} T x^{*}\right\|+\left(1-q_{n}\right)\left\|Q_{\Omega_{n+1}} T y_{n}-Q_{\Omega} T y_{n}\right\| .
\end{gathered}
$$


Since $\left\{T y_{n}\right\}$ is bounded and due to Lemma 2.10, there exist positive constants $M_{5}$ and $M_{6}$ such that

$$
\left\|Q_{\Omega_{n+1}} T y_{n}-Q_{\Omega} T y_{n}\right\| \leq M_{5} \sqrt{h_{X}\left(M_{6} \sigma_{n}\right)} .
$$

This implies

$$
\left\|y_{n+1}-x^{*}\right\| \leq q_{n}\left\|z_{0}-x^{*}\right\|+\left(1-q_{n}\right)\left\|y_{n}-x^{*}\right\|+M_{5} \sqrt{h_{X}\left(M_{6} \sigma_{n}\right)} .
$$

Denoting $\lambda_{n}=\left\|y_{n}-x^{*}\right\|$ we obtain

$$
\lambda_{n+1} \leq\left(1-q_{n}\right) \lambda_{n}+q_{n}\left\|z_{0}-x^{*}\right\|+M_{5} \sqrt{h_{X}\left(M_{6} \sigma_{n}\right)} .
$$

From Lemma 2.11 it follows that $\left\{y_{n}-x^{*}\right\}$ is bounded. Let $\left\|y_{n}-x^{*}\right\| \leq C_{1}$ for all $n$.

Next we evaluate the following difference:

$$
\begin{gathered}
y_{n+1}-y_{n}=\left(1-q_{n}\right)\left(Q_{\Omega_{n+1}} T y_{n}-Q_{\Omega_{n}} T y_{n-1}\right)+\left(q_{n}-q_{n-1}\right)\left(z_{0}-x^{*}\right) \\
+\left(q_{n-1}-q_{n}\right)\left(Q_{\Omega_{n}} T y_{n-1}-Q_{\Omega} T x^{*}\right) \\
=\left(1-q_{n}\right)\left(Q_{\Omega_{n+1}} T y_{n}-Q_{\Omega_{n+1}} T y_{n-1}\right)+\left(1-q_{n}\right)\left(Q_{\Omega_{n+1}} T y_{n-1}-Q_{\Omega_{n}} T y_{n-1}\right) \\
+\left(q_{n}-q_{n-1}\right)\left(z_{0}-x^{*}\right)+\left(q_{n-1}-q_{n}\right)\left(Q_{\Omega_{n}} T y_{n-1}-Q_{\Omega_{n}} T x^{*}\right) \\
+\left(q_{n-1}-q_{n}\right)\left(Q_{\Omega_{n}} T x^{*}-Q_{\Omega} T x^{*}\right) .
\end{gathered}
$$

Using the following estimates

$$
\begin{gathered}
\left\|Q_{\Omega_{n+1}} T y_{n}-Q_{\Omega_{n+1}} T y_{n-1}\right\| \leq\left\|y_{n}-y_{n-1}\right\| \\
\left\|Q_{\Omega_{n}} T y_{n-1}-Q_{\Omega_{n}} T x^{*}\right\| \leq\left\|y_{n-1}-x^{*}\right\| \\
\left\|Q_{\Omega_{n}} T x^{*}-Q_{\Omega} T x^{*}\right\| \leq M_{7} \sqrt{h_{X}\left(M_{8} \sigma_{n}\right)}
\end{gathered}
$$

and

$$
\left\|Q_{\Omega_{n+1}} T y_{n-1}-Q_{\Omega_{n}} T y_{n-1}\right\| \leq M_{9} \sqrt{h_{X}\left(M_{10} \sigma_{n}\right)},
$$

for suitable constants $M_{7}, \ldots, M_{10}$ (see Lemma 2.10), we obtain

$$
\begin{gathered}
\left\|y_{n+1}-y_{n}\right\| \leq\left(1-q_{n}\right)\left\|y_{n}-y_{n-1}\right\|+\left(1-q_{n}\right) M_{9} \sqrt{h_{X}\left(M_{10} \sigma_{n}\right)} \\
\left.+\left|q_{n}-q_{n-1}\right|\left(\left\|z_{0}-x^{*}\right\|+C_{1}\right)+M_{7} \sqrt{h_{X}\left(M_{8} \sigma_{n}\right)}\right) .
\end{gathered}
$$

Denoting $\lambda_{n}=\left\|y_{n}-y_{n-1}\right\|$ one has

$$
\lambda_{n+1} \leq\left(1-q_{n}\right) \lambda_{n}+\gamma_{n},
$$

where

$$
\begin{gathered}
\gamma_{n}=\left|q_{n}-q_{n-1}\right|\left(|| z_{0}-x^{*} \|+C_{1}\right)+\left|q_{n}-q_{n-1}\right| M_{7} \sqrt{h_{X}\left(M_{8} \sigma_{n}\right)} \\
+\left(1-q_{n}\right) M_{9} \sqrt{h_{X}\left(M_{10} \sigma_{n}\right)} .
\end{gathered}
$$


Now, by (5.3), (5.4) and Lemma 2.11, we conclude that $\left\|y_{n}-y_{n-1}\right\| \rightarrow 0$.

Next we show that

$$
\lim _{n \rightarrow \infty}\left\|y_{n}-Q_{\Omega} T y_{n}\right\|=0 .
$$

Indeed,

$$
\begin{aligned}
\left\|y_{n}-Q_{\Omega} T y_{n}\right\| \leq & \left\|y_{n}-Q_{\Omega_{n}} T y_{n-1}\right\|+\left\|Q_{\Omega_{n}} T y_{n-1}-Q_{\Omega} T y_{n-1}\right\| \\
& +\left\|Q_{\Omega} T y_{n-1}-Q_{\Omega} T y_{n}\right\| \\
\leq & \left\|y_{n}-Q_{\Omega_{n}} T y_{n-1}\right\|+M_{7} \sqrt{h_{X}\left(M_{8} \sigma_{n}\right)}+\left\|y_{n-1}-y_{n}\right\|,
\end{aligned}
$$

which states our claim since $\left\|y_{n}-y_{n-1}\right\| \rightarrow 0$ and $\sqrt{h_{X}\left(M_{8} \sigma_{n}\right)} \rightarrow 0$ as $n \rightarrow \infty$, and

$$
\left\|y_{n}-Q_{\Omega_{n}} T y_{n-1}\right\|=q_{n}\left(z_{0}-Q_{\Omega_{n} T y_{n-1}}\right)
$$

which, from the boundedness of $\left\{Q_{\Omega_{n} T y_{n-1}}\right\}$, also tends to 0 .

Now we write

$$
y_{n+1}-Q z_{0}=\left(1-q_{n}\right)\left(Q_{\Omega_{n+1}} T y_{n}-Q z_{0}\right)+q_{n}\left(z_{0}-Q z_{0}\right),
$$

and apply the subdifferential inequality to $J_{\psi}$ as in Theorem 3.1 to deduce that

$$
\phi\left(\left\|y_{n+1}-Q z_{0}\right\|\right) \leq \phi\left(\left(1-q_{n}\right)\left\|Q_{\Omega_{n+1}} T y_{n}-Q z_{0}\right\|\right)+q_{n}\left\langle z_{0}-Q z_{0}, J_{\psi}\left(y_{n+1}-Q z_{0}\right)\right\rangle .
$$

Since $Q z_{0} \in \mathcal{N}\left(Q_{\Omega} T\right) \subseteq \Omega$,

$$
\begin{gathered}
\left\|Q_{\Omega_{n+1}} T y_{n}-Q z_{0}\right\| \leq\left\|Q_{\Omega_{n+1}} T y_{n}-Q_{\Omega_{n+1}} T Q z_{0}\right\|+\left\|Q_{\Omega_{n+1}} T Q z_{0}-Q \Omega T Q z_{0}\right\| \\
\leq\left\|y_{n}-Q z_{0}\right\|+\nu_{n}
\end{gathered}
$$

where $\nu_{n}=M_{7} \sqrt{h_{X}\left(M_{8} \sigma_{n+1}\right)}$ is bounded and vanishes as $n \rightarrow \infty$. From (5.4) we can follow the same reasoning as in Theorem 3.1 to obtain (for $n$ large enough)

$$
\begin{gathered}
\phi\left(\left\|y_{n+1}-Q z_{0}\right\|\right) \leq\left(1-q_{n}\right) \phi\left(\left\|y_{n}-Q z_{0}\right\|\right)+\left(1-q_{n}\right) \nu_{n} M_{11}+ \\
+q_{n}\left\langle z_{0}-Q z_{0}, J_{\psi}\left(y_{n+1}-Q z_{0}\right)\right\rangle .
\end{gathered}
$$

for $M_{11}$ a suitable constant. We claim that

$$
\limsup _{n \rightarrow \infty}\left\langle z_{0}-Q z_{0}, J_{\psi}\left(y_{n}-Q z_{0}\right)\right\rangle \leq 0 .
$$

Since $\left\{y_{n}\right\}$ is bounded there exists a subsequence $\left\{y_{n_{k}}\right\}, y_{n_{k}} \in \Omega_{n_{k}}$ for each $k$, which weakly converges to some point $\bar{y}$ and

$$
\limsup _{n \rightarrow \infty}\left\langle z_{0}-Q z_{0}, J_{\psi}\left(y_{n}-Q z_{0}\right)\right\rangle=\lim _{k \rightarrow \infty}\left\langle z_{0}-Q z_{0}, J_{\psi}\left(y_{n_{k}}-Q z_{0}\right)\right\rangle .
$$

Now we apply the following result: 
Lemma 5.2 [16] If the set $\Omega$ is convex and closed set in reflexive Banach space $X$ and the sequence of sets $\Omega_{n} \subseteq X$ satisfy the limit relation $\mathcal{H}\left(\Omega_{n}, \Omega\right) \rightarrow 0$ as $n \rightarrow \infty$ then every weak limit point $u$ of any sequence $\left\{u_{n}\right\}, u_{n} \in \Omega_{n}$, belongs to the subset $\Omega$.

To deduce that $\bar{y} \in \Omega$. The rest of the proof follows the pattern of Theorem 3.1 once we prove that $\bar{y}$ is a fixed point of $Q_{\Omega} T$. To prove this we make use of Opial's condition used in the following form:

$$
\begin{gathered}
\liminf _{l \rightarrow \infty}\left\|y_{n_{l}}-\bar{y}\right\|<\liminf _{l \rightarrow \infty}\left\|y_{n_{l}}-Q_{\Omega} T \bar{y}\right\| \\
\leq \liminf _{l \rightarrow \infty}\left(\left\|y_{n_{l}}-Q_{\Omega} T y_{n_{l}}\right\|+\left\|Q_{\Omega} T y_{n_{l}}-Q_{\Omega} T \bar{y}\right\|\right) \\
\leq \liminf _{l \rightarrow \infty}\left\|Q_{\Omega} T y_{n_{l}}-Q_{\Omega} T \bar{y}\right\| \leq \liminf _{l \rightarrow \infty}\left\|y_{n_{l}}-\bar{y}\right\| .
\end{gathered}
$$

Remark 5.3 Notice that in this theorem we do not obtain convergence to a fixed point of $T$. Convergence to a fixed point of $T$ can be obtained if we impose certain boundary conditions on $T$ as, for instance, that $T(\partial \Omega) \subseteq \Omega$. It is not hard to see that in this case $\mathcal{N}\left(Q_{\Omega} T\right)=\mathcal{N}(T)$.

Remark 5.4 The sunny nonexpansive retraction onto $\mathcal{N}\left(Q_{\Omega} T\right)$ always exists in this case since $Q_{\Omega} T$ is a nonexpansive self-mapping [8].

Remark 5.5 If the condition $\bar{G}=\Omega \cap G \neq \emptyset$ does not hold we prove the previous theorem in the following way. Instead of $\Omega_{n}$ we consider the collection of sets $\Omega_{n}^{\prime}=\overline{c o}\left(\Omega \cup \Omega_{n}\right)$, where $\overline{c o}(A)$ stands for the closed convex closure of a set $A$. It is easy to see that $\mathcal{H}\left(\Omega, \Omega_{n}^{\prime}\right) \rightarrow 0$ as $n \rightarrow \infty$. Now it suffices to follow the same proof.

Remark 5.6 The sequence $\left\{T y_{n}\right\}$ is bounded if, for instance, $\left\{y_{n}\right\}$ is bounded. This obviously holds if, for instance, $\Omega$ is bounded.

\section{References}

[1] Alber, Ya.I.: Generalized projection operators in Banach spaces: properties and applications. Functional Differential Equations 1, 1-21 (1994).

[2] Alber, Ya.I., Chidume C.E., Zegeye H.: Approximating fixed points of total asymptotically nonexpansive mapings, Fixed Point Theory and Applications 2006, article ID 10673, 1-20.

[3] Alber, Ya.I., Reich, S., Yao Jen-Chih: Iterative methods for solving fixed-point problems with nonself-mappings in Banach spaces. Abstract and Applied Analysis 4, 193-216 (2003).

[4] Alber, Ya.I. and Ryazantseva, I.P., Nonlinear Ill-posed Problems of Monotone Type, Springer, 2006. 
[5] Browder, F.E.: Convergence theorems for sequences of nonlinear operators in Banach spaces. Math. Z. 100, 201-225 (1967).

[6] Chidume, C.E., Li, J., Udomene, A.: Convergence of paths and approximation of fixed points of asymptotically nonexpansive mappings. Proc. Amer. Math. Soc. 133, 473-480 (2005).

[7] Chidume, C.E., Ofoedu, E.U., Zegeye, H.: Strong and weak convergence theorems for asymptotically nonexpansive mappings. J. Math. Anal. Appl. 280, 364-374 (2003).

[8] Domínguez Benavides, T., Lorenzo Ramírez, P.: Structure of the fixed point set and common fixed points of asymptotically nonexpansive mappings, Proc. Amer. Math. Soc. 129 (12), 3549-3557 (2001).

[9] Figiel, T.: On the moduli of convexity and smoothness, Studia Mathematica 56, 121155 (1976).

[10] K. Goebel, K. and Reich, S., Uniform Convexity, Hyperbolic Geometry, and Nonexpansive Mappings, Marcel Dekker, New York and Basel, 1984.

[11] García-Falset, J., Sims, B., Smyth, M.: The demiclosedness principle for mappings of asymptotically nonexpansive type, Houston J. Math. 22, 101-108(1996).

[12] Halpern, B.: Fixed points of non-expanding maps. Bull. Amer. Math. Soc. 73, 957-961 (1967).

[13] Khamsi, M.A., On uniform Opial condition and uniform Kadec-Klee property in Banach and metric spaces. Nonl. Anal. 26, 1733-1748 (1996).

[14] Lin, P.K., Tan, K.K., Xu, H.K.: Demiclosedness principle and asymptotic behaviour for asymptotically nonexpansive mappings. Nonl. Anal. 24, 929-946 (1995).

[15] Lim, T.C., Xu, H.X.: Fixed point theorems for asymptotically nonexpansive mappings. Nonl. Anal. 22, 1345-1355 (1994).

[16] Liskovets, O.A.: Regularization of ill-posed variational inequalities on approximately given sets (Russian). Dokl. Akad. Nauk SSSR 317 (2), 300-304 (1991). (Translation in Soviet Math. Dokl. 43 (2), 384-387 (1991).)

[17] Reich, S.: Strong convergence theorems for resolvents of accretive operators in Banach spaces. J. Math. Anal. Appl. 75, 287-292 (1980).

[18] Reich, S.: Approximating Fixed Points of Nonexpansive Mapings. Panamerican Mathematical Journal 4 23-28 (1994).

[19] Takahashi, W., Kim, G.E.: Strong convergence of approximants to fixed points of nonexpansive nonself-mappings in Banach spaces. Nonlinear Anal. 32, 447-454 (1998).

[20] Wittmann R.: Approximation of fixed points of nonexpansive mappings. Arch. Math. 58, 486-491 (1992). 
[21] Xu, H.K.: Strong convergence of an iterative method for nonexpansive and accretive operators. J. Math. Anal. Appl. 314 (2), 631-643 (2006). 